\title{
Phase-Dependent Chiral Transport and Effective Non-Hermitian Dynamics in a Bosonic Kitaev-Majorana Chain
}

\author{
A. McDonald, ${ }^{1,2}$ T. Pereg-Barnea, ${ }^{1,3}$ and A. A. Clerk $^{2}$ \\ ${ }^{1}$ Department of Physics, McGill University, Montréal, Québec, Canada H3A 2T8 \\ ${ }^{2}$ Institute for Molecular Engineering, University of Chicago, Chicago, Illinois 60637, USA \\ ${ }^{3}$ Department of Condensed Matter Physics, Weizmann Institute of Science, Rehovot, 76100, Israel
}

(Received 7 June 2018; revised manuscript received 22 August 2018; published 21 November 2018)

\begin{abstract}
We study a 1D chain of noninteracting bosonic cavities which are subject to nearest-neighbor parametric driving, thus realizing a bosonic Hamiltonian whose form is reminiscent of the celebrated Kitaev model of a 1D $p$-wave superconductor. For a suitable choice of drive phases, the model exhibits a number of remarkable properties. This includes phase-dependent chirality: Photons propagate and are amplified in a direction determined by the phase of the initial drive or excitation. It also exhibits a drastic sensitivity to boundary conditions: For a range of parameters, the boundaryless system has only delocalized, dynamically unstable modes, while a finite open chain is described by localized, dynamically stable modes. While our model is described by a Hermitian Hamiltonian, we show that it has a surprising connection to non-Hermitian asymmetric hopping models. In addition to being of fundamental interest as a new kind of topological bosonic system, our system also has potential practical utility as a quantum amplifier and a source of multimode entangled photons.
\end{abstract}

DOI: 10.1103/PhysRevX.8.041031

Subject Areas: Condensed Matter Physics, Photonics, Quantum Information

\section{INTRODUCTION}

Superconducting fermionic systems are by now well known to exhibit unique kinds of topological behavior. Perhaps the best-known example is the Kitaev chain model [1], the simplest possible model of a spinless $p$-wave superconductor: One takes a 1D tight-binding chain of spinless fermions with nearest-neighbor hopping and adds pairing terms on each bond. This model exhibits topologically protected end Majorana zero modes and underpins the current quest to realize Majorana-based topological quantum computation [2-6].

Parametrically driven bosonic systems have quadratic Hamiltonians with the same basic form as that of a fermionic superconductor. The parametric drive corresponds to coherent two-photon addition and removal and is analogous to a superconducting pair potential. The lack of any exclusion principle implies that such bosonic models can differ strongly from their fermionic counterparts. Recent work has demonstrated that noninteracting parametrically driven models can realize unique forms of topological phases, with bands characterized by an integer

Published by the American Physical Society under the terms of the Creative Commons Attribution 4.0 International license. Further distribution of this work must maintain attribution to the author(s) and the published article's title, journal citation, and DOI.
Chern number, and with edge states that act as ideal amplifying or squeezing channels [7-14]. Work has also shown that in 1D, strong interactions fermionize bosons, letting parametric drives directly realize many aspects of the fermionic Kitaev chain $[15,16]$.

(a)

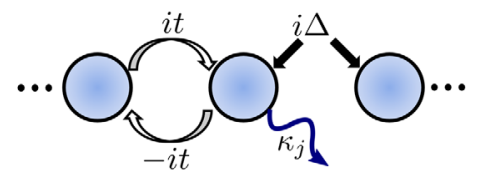

(b)

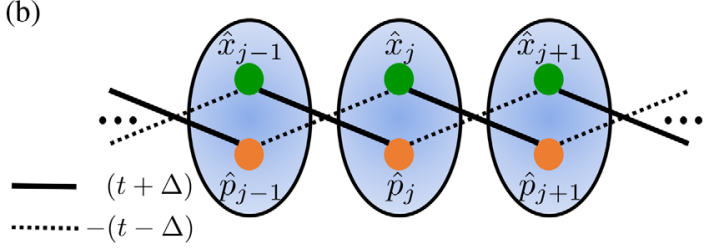

FIG. 1. (a) Schematic of the setup: An array of tunnel-coupled cavities (hopping $t$, on-site loss $\kappa_{j}$ ) is subject to resonant parametric driving (amplitude $\Delta$ ) on each bond. We require a purely imaginary hopping matrix element, which is gauge equivalent to having staggered parametric drive phases. (b) When written in terms of local cavity quadratures $\hat{x}_{j}$ and $\hat{p}_{j}$, the model describes a spatially asymmetric pattern of couplings reminiscent of the asymmetric coupling of Majorana operators in the fermionic Kitaev-Majorana chain. The system exhibits phasedependent chiral transport that is robust against arbitrary spatially varying loss. 
In this work, we consider a one-dimensional, parametrically driven system whose quadratic Hamiltonian has an almost identical form to the fermionic Kitaev chain. Unlike Ref. [15], we do not require strong on-site repulsive interactions but instead consider the effects of reducing the symmetry of the system by introducing hopping phases. Remarkably, we find that this system has striking features reminiscent of the Kitaev chain: The system is best understood in terms of Hermitian quadrature operators, not photonic annihilation operators, and exhibits a strong sensitivity to boundary conditions, including the presence of highly localized states. The system also has unique transport properties: Photons propagate and are amplified in a chiral fashion, with the direction being set by the phase of the initial excitation, which is in stark contrast to more conventional traveling-wave amplifiers. We also point out a surprising connection between the dynamics of our model and non-Hermitian asymmetric hopping models [17].

We believe that our system is of fundamental interest in that it represents a new kind of topological bosonic system. It also has potential in a variety of quantum-information and quantum-photonics settings. The phase-sensitive chiral amplification represents a new kind of quantum amplifier, one that could be used for the readout and routing of quantum information. In addition, we discuss how the system could be used to generate complex multimode entangled Gaussian states in a manner that is simply related to a network of beam splitters driven by input squeezing. Such states have garnered recent attention as a means of demonstrating quantum supremacy and for applications ranging from simulating the vibronic spectra of molecules [18] to solving graph-theoretic problems [19].

In what follows, we introduce our model, outline its exceptional properties, and discuss implementations based on superconducting quantum circuits.

\section{MODEL}

We start by recalling the fermionic Kitaev model [1]: spinless electrons on a 1D lattice subject to a $p$-wave pairing potential. In momentum space and at zero chemical potential, it takes the form

$$
\hat{H}_{F}=\sum_{k}\left[t \cos k \hat{c}_{k}^{\dagger} \hat{c}_{k}+i \frac{\Delta}{2} \sin k\left(\hat{c}_{k}^{\dagger} \hat{c}_{-k}^{\dagger}-\text { H.c. }\right)\right]
$$

where $\hat{c}_{k}$ is a fermionic annihilation operator, and the sum runs over the first Brillouin zone; we take $t>0, \Delta>0$ throughout. The remarkable features of this model can be related to its topological properties. To see this, we define $C_{k}^{\dagger}=\left(\hat{c}_{k}^{\dagger}, \hat{c}_{-k}\right)$ and write Eq. (1) as

$$
\hat{H}_{F}=\frac{1}{2} \sum_{k} C_{k}^{\dagger}\left[\mathbf{h}_{F}(k) \cdot \check{\boldsymbol{\sigma}}\right] C_{k},
$$

where $\mathbf{h}_{F}(k)=[0,-\Delta \sin (k), t \cos (k)]$, and $\check{\boldsymbol{\sigma}}$ is a vector of Pauli matrices in particle-hole space. The Hamiltonian $\hat{H}_{F}$ has chiral symmetry as the first component of the vector $\mathbf{h}_{F}(k)$ is zero for all momenta which allows us to define a topological number associated with the number of times that $\mathbf{h}_{F}(k)$ encircles the origin. In the Kitaev model, the topological number is nonzero as the vector $\mathbf{h}_{F}(k)$ winds once.

Can we construct a similar bosonic model with momentum space winding? At first glance, the answer is no. Simply replacing fermionic operators in Eq. (1) with bosonic ones does not work: Since bosonic operators commute (not anticommute), the two-photon term must now be an even function of $k$ and thus cannot be proportional to $\sin k$. As a result, a bosonic model with the identical real-space form as the fermionic Kitaev chain has no nontrivial topology and no unusual properties (see Appendix A).

To obtain a bosonic model that has a nonzero winding in momentum space, we can keep the pairing term even in $k$ but instead make the kinetic energy vary as $\sin k$ :

$$
\hat{H}_{B}=\sum_{k}\left[t \sin k \hat{a}_{k}^{\dagger} \hat{a}_{k}+i \frac{\Delta}{2} \cos k\left(\hat{a}_{k}^{\dagger} \hat{a}_{-k}^{\dagger}-\text { H.c. }\right)\right],
$$

where $\mathbf{h}_{F}(k)$ from the fermionic case is replaced by $\mathbf{h}_{B}(k)=(0,-\Delta \cos k, t \sin k)$, which also winds once around the origin. The form of $\hat{H}_{B}$ in real space

$$
\hat{H}_{B}=\frac{1}{2} \sum_{j}\left(i t \hat{a}_{j+1}^{\dagger} \hat{a}_{j}+i \Delta \hat{a}_{j+1}^{\dagger} \hat{a}_{j}^{\dagger}+\text { H.c. }\right)
$$

is almost identical to that of the Kitaev chain, except that we now have a purely imaginary hopping matrix element. Because of the nonzero pair potential, this $\pi / 2$ phase cannot be completely gauged away; the best one can do is make the Hamiltonian real, at the expense of doubling the size of the unit cell [see Eq. (29) below]. As we now explore in detail, this model has a number of remarkable properties. The case of an arbitrary phase for the hopping matrix element is treated in Appendix B; for a range of parameters, such models exhibit analogous physics to the model in Eq. (4).

\section{QUADRATURE REPRESENTATION}

Recall that the fermionic Kitaev chain is best understood in terms of Hermitian Majorana fermion operators, as opposed to the original (Dirac) fermions [2]. The Majorana operators are defined via $\hat{c}_{j}=e^{i(\pi / 4)}\left(\hat{\gamma}_{j}^{A}+i \hat{\gamma}_{j}^{B}\right) / 2$. When the Hamiltonian in Eq. (1) is expressed using these operators, one finds that $A$-type Majoranas couple only to $B$-type Majoranas on neighboring sites. Moreover, this coupling is spatially asymmetric. This unusual pairing directly leads to the existence of unpaired Majorana end modes in an open chain. 
Remarkably, the bosonic model in Eq. (4) exhibits an analogous structure when we express it in terms of Hermitian quadrature operators $\hat{x}_{j}, \hat{p}_{j}$ on each site, defined via $\hat{a}_{j}=\left(\hat{x}_{j}+i \hat{p}_{j}\right) / \sqrt{2}$. A direct substitution yields

$$
\hat{H}_{B} \equiv \frac{1}{2} \sum_{j}\left[-(t-\Delta) \hat{x}_{j+1} \hat{p}_{j}+(t+\Delta) \hat{p}_{j+1} \hat{x}_{j}\right] .
$$

The structure here is analogous to the fermionic case: $\hat{x}$ quadratures are coupled only to $\hat{p}$ quadratures, and further, there is an asymmetry in the coupling between $\hat{x}_{j}$ and $\hat{p}_{j \pm 1}$ (see Fig. 1).

In the fermionic Kitaev chain, the system is gapped: The hopping asymmetry leads to isolated Majorana modes on the edges, while the bulk can carry only supercurrent. Conversely, the asymmetric coupling in the bosonic version gives rise to unusual propagation within the bulk which has no fermionic counterpart. Indeed, perhaps the most dramatic consequence of the quadrature pairing structure in Eq. (5) is in the dynamics. The Heisenberg equations of motion corresponding to $\hat{H}_{B}$ (with $\hbar=1$ throughout) are

$$
\begin{aligned}
& \dot{\hat{x}}_{j}=\frac{t+\Delta}{2} \hat{x}_{j-1}-\frac{t-\Delta}{2} \hat{x}_{j+1}, \\
& \dot{\hat{p}}_{j}=\frac{t-\Delta}{2} \hat{p}_{j-1}-\frac{t+\Delta}{2} \hat{p}_{j+1} .
\end{aligned}
$$

We see that the dynamics of the $\hat{x}$ quadratures are completely independent of the $\hat{p}$ quadratures. Further, each quadrature is forced in a spatially asymmetric manner by its neighbors. As $t$ approaches $\Delta$, we have complete asymmetry: $\hat{x}$ quadratures are forced only by their neighbors to the left, and $\hat{p}$ quadratures are forced only by their neighbors to the right. Viewing quadratures as particles, we thus expect chiral propagation: $x$ "particles" would propagate only to the right and $p$ particles only to the left. As we will see, this basic expectation is borne out: Eqs. (6) and (7) imply that the transport of a photonic wave packet in our chain will be directional, with the direction determined by the phase of the wave packet. This behavior is reminiscent of edge states in the quantum spin Hall effect, where directionality of an edge state is determined by its spin [20-22].

Before proceeding, it is important to ask whether our system is dynamically stable: Can $\hat{H}_{B}$ be diagonalized? Unlike a fermionic system, the pairing terms in Eq. (5) can lead to dynamical instability, analogous to a standard parametric instability. Focusing on a finite chain with openboundary conditions, we find that the system is stable as long as $t>\Delta$, independent of the chain length. In contrast, the case $t<\Delta$ is always dynamically unstable (see Appendix C). We thus focus exclusively on the case $t>\Delta$ throughout the manuscript. In this regime, the Hamiltonian is unitarily equivalent to a simple tightbinding chain with no parametric drive, as we now show.
Defining the parameter $r$ via

$$
e^{2 r}=(t+\Delta) /(t-\Delta),
$$

we consider a position-dependent local squeezing transformation defined by

$$
\hat{U} \hat{x}_{j} \hat{U}^{\dagger}=e^{r\left(j-j_{0}\right)} \hat{\tilde{x}}_{j}, \quad \hat{U} \hat{p}_{j} \hat{U}^{\dagger}=e^{-r\left(j-j_{0}\right)} \hat{\tilde{p}}_{j} .
$$

Here, $\hat{\tilde{x}}_{j}, \hat{\tilde{p}}_{j}$ are new canonical quadratures, and $j_{0}$ is an arbitrary real number. One finds that

$$
\begin{aligned}
\hat{U} \hat{H}_{B} \hat{U}^{\dagger} & =\frac{\tilde{t}}{2} \sum_{j}\left(-\hat{\tilde{x}}_{j+1} \hat{\tilde{p}}_{j}+\hat{\tilde{p}}_{j+1} \hat{\tilde{x}}_{j}\right) \\
& =\frac{1}{2} \sum_{j}\left(i \tilde{i} \hat{\tilde{a}}_{j+1}^{\dagger} \hat{\tilde{a}}_{j}+\text { H.c. }\right),
\end{aligned}
$$

with

$$
\tilde{t}=\sqrt{t^{2}-\Delta^{2}}=t / \cosh (r) .
$$

Thus, in the stable regime $t>\Delta$, the system is unitarily equivalent to a simple (excitation-conserving) tight-binding chain with a hopping matrix element $i \tilde{t}$. Note that the transformed Hamiltonian is completely independent of the parameter $j_{0}$. This reflects the invariance of $\hat{H}_{B}$ under any spatially uniform squeezing transformation that does not mix $\hat{x}$ and $\hat{p}$ quadratures, i.e., $\hat{x}_{j} \rightarrow e^{z} \hat{x}_{j}, \hat{p}_{j} \rightarrow e^{-z} \hat{p}_{j}$.

We also briefly comment on the special case $t=\Delta$. In this case, Eq. (5) implies a complete coupling asymmetry: $\hat{x}_{j}$ is coupled only to $\hat{p}_{j+1}$ (and not to $\hat{p}_{j-1}$ ). For an open chain with $N$ sites, there are thus two localized quadrature operators at the ends of the chain, $\hat{p}_{1}$ and $\hat{x}_{N}$, that drop out of the Hamiltonian. While in the fermionic model, the corresponding decoupled modes are of great interest, they are of less interest here. Unlike with fermions, these decoupled quadrature operators cannot be recombined to form a new, delocalized canonical bosonic mode: Since $\hat{x}_{N}$ and $\hat{p}_{1}$ commute, any operator $\hat{f}$ which is a linear combination of both quadratures will never satisfy the bosonic commutation relation $\left[\hat{f}, \hat{f}^{\dagger}\right]=\hat{1}$. While these decoupled quadratures do represent quantum nondemolition observables, their existence here requires precise tuning to the threshold of instability.

\section{PHASE-SENSITIVE CHIRAL PROPAGATION}

Equations (6) and (7) imply that photonic excitations in our lattice propagate in a chiral fashion, with a direction that depends on the phase of the excitation. To fully characterize this behavior, we calculate the retarded single-particle Green's functions for our system in position space, focusing on an $\mathrm{N}$-site open chain. To make the chirality explicit, we calculate quadrature-quadrature 
Green's functions. This is easily done using the positiondependent squeezing transformation in Eq. (9). One finds that the only nonzero Green's functions are

$$
\begin{aligned}
G_{x}^{R}\left[j, j^{\prime} ; \omega\right] & \equiv-i \int_{0}^{\infty} d t e^{i \omega t}\left\langle\left[\hat{x}_{j}(t), \hat{p}_{j^{\prime}}(0)\right]\right\rangle \\
& =i \tilde{G}_{0}^{R}\left[j, j^{\prime} ; \omega\right] e^{r\left(j-j^{\prime}\right)}, \\
G_{p}^{R}\left[j, j^{\prime} ; \omega\right] & =-i \tilde{G}_{0}^{R}\left[j, j^{\prime} ; \omega\right] e^{-r\left(j-j^{\prime}\right)} .
\end{aligned}
$$

Here, $\tilde{G}_{0}^{R}\left[j, j^{\prime} ; \omega\right]$ is the retarded-photon Green's function of an $N$-site tight-binding chain with tunnel matrix element $i \tilde{t}$ (see Appendix D).

The Green's function $G_{x}^{R}\left[j, j^{\prime} ; \omega\right]$ describes propagation of the $x$ quadrature in the lattice; more explicitly, it describes how $\hat{x}_{j}$ responds to a perturbation which directly forces $\hat{x}_{j^{\prime}} . G_{p}^{R}\left[j, j^{\prime} ; \omega\right]$ is interpreted analogously. The Green's functions above directly manifest the expected chirality: $x$ quadrature signals are amplified (deamplified) as they propagate from left to right (right to left); the $p$ quadratures exhibit the opposite behavior. Note that the local Green's functions $\left(j=j^{\prime}\right)$ have no explicit $r$ dependence or phase sensitivity: They are identical to those of a particle-conserving tight-binding model with hopping $i \tilde{t}$.

Remarkably, the above structure still holds if we break translational invariance by introducing position-dependent loss on each site. We treat this loss as Markovian and model it using standard input-output theory [23,24]. The loss gives rise to a damping rate $\kappa_{j}$ on each lattice site; it could be due to a deliberate coupling to waveguides or to internal loss. The Heisenberg-Langevin equations for the $\hat{x}$ and $\hat{p}$ quadratures on each site now read

$$
\begin{aligned}
& \dot{\hat{x}}_{j}=\frac{\tilde{t}}{2}\left(e^{r} \hat{x}_{j-1}-e^{-r} \hat{x}_{j+1}\right)-\frac{1}{2} \kappa_{j} \hat{x}_{j}-\sqrt{\kappa_{j}} \hat{x}_{j}^{(\mathrm{in})}, \\
& \dot{\hat{p}}_{j}=\frac{\tilde{t}}{2}\left(e^{-r} \hat{p}_{j-1}-e^{r} \hat{p}_{j+1}\right)-\frac{1}{2} \kappa_{j} \hat{p}_{j}-\sqrt{\kappa_{j}} \hat{p}_{j}^{(\mathrm{in})} .
\end{aligned}
$$

$\hat{x}_{j}^{(\mathrm{in})}(t)$ and $\hat{p}_{j}^{(\mathrm{in})}(t)$ describe the quadratures of the input field associated with the loss port $\kappa_{j}$; in the simplest case, they just describe vacuum fluctuations.

Despite the additional terms due to loss, we can still map our system to a simple tight-binding model with no pairing terms. One simply applies the local-site-dependent squeezing transformation in Eq. (9) to the Langevin equations. After this transformation, the Heisenberg-Langevin equations describe a simple tight-binding model with renormalized hopping $\tilde{t}$ [cf. Eq. (11)] and decay rate $\kappa_{j}$ on each site. While the input noise operators now describe squeezed noise, the linearity of the equations means that this does not have any effect on the Green's functions. As a result, even with loss, the Green's functions of our system still have the form of Eqs. (12) and (13), where now $\tilde{G}_{0}^{R}\left[j, j^{\prime} ; \omega\right]$ is the photonic Green's function of a tight-binding chain with hopping $\tilde{t}$ and on-site loss rates $\kappa_{j}$ (see Appendix D). Note that this squeezing transformation still maps the model to a particle-conserving one since $x$ and $p$ quadratures remain dynamically decoupled in the presence of on-site loss. In Sec. VII, we discuss other relevant perturbations which couple these orthogonal quadratures.

Finally, we stress that the phase-dependent chirality manifested by the Green's functions also reflects itself in simple wave-packet dynamics. To be concrete, suppose we initially prepare our lattice in a coherent-state wave packet with zero average momentum. Such a state is characterized by $\left\langle\hat{a}_{j}(0)\right\rangle=e^{i \theta} f_{j}$, where $\theta$ is the global phase of the excitation, and $f_{j}>0$ describes the envelope of the wave packet. We can now directly use Eqs. (6) and (7) to determine the evolution of $\left\langle\hat{a}_{j}(t)\right\rangle$ and hence the motion of the wave packet. We see that the wave packet will split into two: The $\cos \theta$ component of the wave packet corresponds to an $x$ quadrature excitation that propagates and is amplified as it moves to the right, while the $\sin \theta$ component is a $p$ quadrature excitation that propagates to the left.

\section{SCATTERING PROPERTIES}

We now consider the case where input-output waveguides are attached to our lattice and ask how fields incident on the lattice from these waveguides are scattered. The relevant scattering matrix follows immediately from the input-output boundary condition $\hat{a}_{j}^{\text {(out) }}=\hat{a}_{j}^{\text {(in) }}+\sqrt{\kappa_{j}} \hat{a}_{j}$ $[23,24]$ and the Heisenberg-Langevin equations in Eqs. (14) and (15). As $x$ and $p$ quadratures are dynamically decoupled in our system, scattering does not mix these quadratures. The scattering matrix thus takes a simple form in the quadrature basis and is defined by

$$
\begin{aligned}
& \hat{x}_{j}^{(\text {out })}[\omega]=\sum_{j^{\prime}} s_{j j^{\prime}}^{x}[\omega] \hat{x}_{j^{\prime}}^{\text {(in) }}[\omega], \\
& \hat{p}_{j}^{\text {(out) })}[\omega]=\sum_{j^{\prime}} s_{j j^{\prime}}^{p}[\omega] \hat{p}_{j^{\prime}}^{\text {(in) }}[\omega] .
\end{aligned}
$$

The scattering matrix is directly determined by the system Green's functions and thus inherits their structure:

$$
\begin{gathered}
s_{j j^{\prime}}^{x}[\omega]=e^{r\left(j-j^{\prime}\right)} \tilde{s}_{j j^{\prime}}[\omega], \\
s_{j j^{\prime}}^{p}[\omega]=e^{-r\left(j-j^{\prime}\right)} \tilde{s}_{j j^{\prime}}[\omega] .
\end{gathered}
$$

Here, $\tilde{s}_{j j^{\prime}}[\omega]$ is the scattering matrix of an $N$-site tightbinding chain with tunnel matrix element $i \tilde{t}$ and decay rate $\kappa_{j}$ on each site (see Appendix D); the scattering in such a system is insensitive to phase, and hence the same for any quadrature. We see that in our full system, as expected, the $\hat{x}$ quadrature is amplified (deamplified) in transmission 
(a)

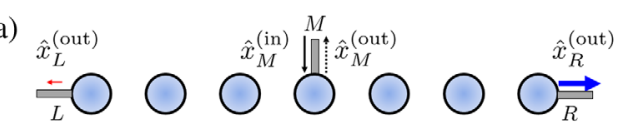

(b)

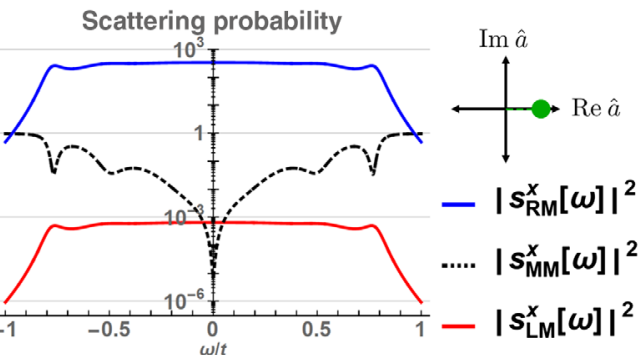

(c)

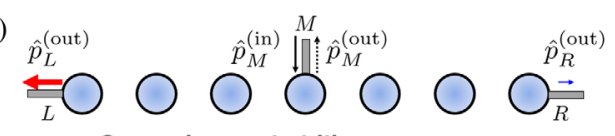

(d)

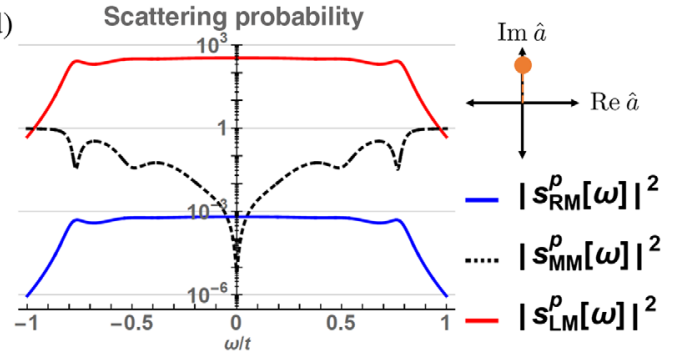

FIG. 2. Scattering properties of the bosonic Kitaev-Majorana chain. (a) Schematic of the setup. The leftmost, middle, and rightmost sites are attached to waveguides (coupling rates $\kappa_{L}, \kappa_{M}$, and $\kappa_{R}$, respectively). A signal with a frequency $\omega$ and global phase $\theta=0$ corresponding to an $x$ excitation is injected in the middle waveguide and is amplified (deamplified) as it propagates to the right (left). (b) Amplitude squared of the scattering matrix elements plotted as a function of the frequency of the input signal. As expected, signals propagating to the right (left) are amplified (deamplified). Note the reflection probability (black) is bounded by unity. (c) Same setup as in (a), except the phase of the signal is now $\theta=\pi / 2$ corresponding to a $p$ excitation. (d) The signal is now amplified (deamplified) as it propagates to the left (right). For (b),(d), we take $N=13$ sites, $\Delta=t / 2$, uniform on-site internal loss rate $\kappa=10^{-2} t$, and waveguide couplings $\kappa_{M}=2 \kappa_{L}=2 \kappa_{R}=2 t$.

from left to right (right to left), whereas the $\hat{p}$ quadrature exhibits the opposite behavior (see Fig. 2).

Our system thus represents a unique kind of phasesensitive amplifier. Such devices amplify only one quadrature of an input signal (deamplifying the other conjugate quadrature) and are capable of quantum amplification with zero added noise [24]. They also serve as sources of nonclassical quadrature squeezed light. Standard phasesensitive amplifiers either amplify only in one direction (due to phase matching) or are reciprocal and amplify the same quadrature irrespective of transmission direction. In contrast, we obtain amplification in both directions but in different orthogonal quadratures. Note that the end-to-end transmission gains $\left|s_{N 1}^{x}[\omega]\right|^{2}=\left|s_{1 N}^{p}[\omega]\right|^{2}$ scale like $e^{2 r N}$, while the amplification bandwidth scales like $\tilde{t}=$ $t / \cosh (r)$. Our system is thus not limited by a standard gain-bandwidth product: By using a long chain and a small $r$, large gain is possible without sacrificing bandwidth.

Viewed as an amplifier, our system has another remarkable property: While there is strong gain in transmission, there is never any gain in reflection. This follows immediately from Eqs. (17) and (18), which tells us that $s_{j j}^{x}[\omega]=$ $s_{j j}^{p}[\omega]=\tilde{s}_{j j}[\omega]$ : The reflection amplitude coincides with that of a simple tight-binding model with hopping $i \tilde{i}$, and hence it can never be larger than unity. The lack of reflection gain is of practical utility in many settings, where one wishes to protect a fragile signal source coupled to an input port.

For an intuitive picture of the lack of reflection gain, consider trajectories and the equations of motion in Eqs. (6) and (7). Any reflection process requires an equal amount of left-to-right propagation and right-to-left propagation. In our system, the directional nature of the amplification means that the net amplification for such a process will always be zero: Amplification in one direction is perfectly compensated by deamplification in the opposite direction.

\section{EXTREME SENSITIVITY TO THE PRESENCE OF EDGES}

A key feature of the fermionic Kitaev-Majorana chain is a marked sensitivity to the presence of edges: For periodic boundary conditions, the system has an energy gap centered around zero energy, whereas with open-boundary conditions, there exist localized, zero-energy Majorana edge modes. We find that our bosonic analogue of the Kitaev chain also exhibits an extreme sensitivity to the presence of edges: With periodic boundary conditions, the system is always characterized by unstable, spatially extended eigenmodes, whereas with open-boundary conditions, it can be completely stable (all mode energies real), and further, it can have completely localized wave functions. We explain this remarkable behavior further in what follows.

Consider first the system with periodic boundary conditions and no dissipation. The Heisenberg equations of motion in momentum space take the form

$$
\begin{aligned}
& \left(i \partial_{t}-\check{M}_{k}\right)\left(\begin{array}{c}
\hat{a}_{k} \\
\hat{a}_{-k}^{\dagger}
\end{array}\right)=\left(\begin{array}{l}
0 \\
0
\end{array}\right), \\
& \check{M}_{k}=t \sin (k) \check{1}+i \Delta \cos (k) \check{\sigma}_{x} .
\end{aligned}
$$

As usual, the mode energies are the eigenvalues of the dynamical matrix $\check{M}_{k}$ and are given by $E_{k, \pm}=t \sin (k) \pm$ $i \Delta \cos (k)$. The fact that the eigenvalues are complex for all $k$ (except $\pm \pi / 2$ ) indicates that for any nonzero $\Delta$, the system is past the threshold for parametric instability and will exhibit exponential growth in the time domain. This is 
not surprising, as the parametric drive is resonant: The drive is adding pairs of photons with zero total momentum, and the energy detuning of such a pair is always zero. Note that the wave functions of these modes are simple plane waves, consistent with the translational invariance of the model.

While intuitively reasonable, the behavior of the periodic boundary condition system is in stark contrast to the open-boundary-condition system. As already shown in Eq. (9), in this case we can make a unitary squeezing transformation for any $t>\Delta$ to map our system to a simple tight-binding model with hopping matrix element $i \tilde{t}=i \sqrt{t^{2}-\Delta^{2}}$. As such, there is no instability: All mode eigenvalues are real, given by $E_{n}=\tilde{t} \cos k_{n}$, with $k_{n}=n \pi /(N+1)$. We thus have a dramatic difference in the spectrum of the model depending on if the system has edges or not (see Fig. 3). Note that this conclusion is independent of system size.

The difference between a ring and chain geometry goes beyond just the spectrum: The wave functions are also completely different in the two cases. Naively, one would expect that for the open chain, the eigenstates are simple standing waves formed by taking linear combinations of the plane-wave eigenstates of the ring. This is not the case. For an $N$-site open chain, the diagonalized Hamiltonian can be written as $\hat{H}_{B}^{\mathrm{OBC}}=\sum_{n} E_{n} \hat{\beta}_{n}^{\dagger} \hat{\beta}_{n}$. The quasiparticle $\hat{\beta}_{n}$ is given by a Bogoliubov transformation of our original realspace photon operators,

$$
\hat{\beta}_{n}=\sum_{j=1}^{N}\left[u_{n}(j) \hat{a}_{j}-v_{n}(j) \hat{a}_{j}^{\dagger}\right],
$$

with the functions $u_{n}(j), v_{n}(j)$ representing the "particle" and "antiparticle" parts of the wave function. Using the squeezing transformation in Eq. (9) which diagonalizes our Hamiltonian, one finds easily

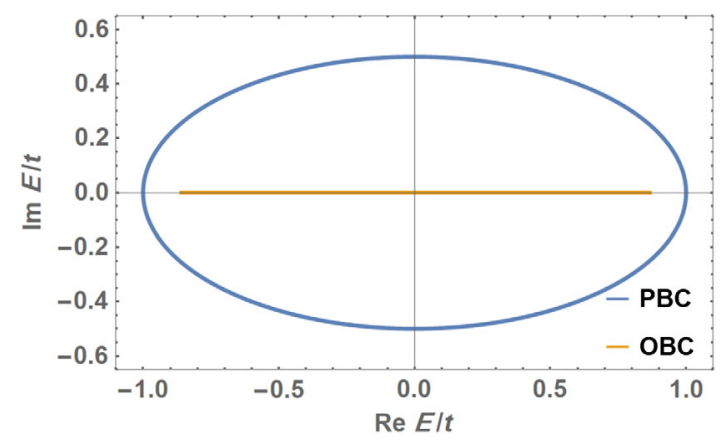

FIG. 3. Spectrum of the system with periodic boundary condition (PBC) $E_{k, \pm}^{\mathrm{PBC}}=t \sin (k) \pm i \Delta \cos (k)$ versus open-boundary conditions (OBC) $E_{k}^{\mathrm{OBC}}=\sqrt{t^{2}-\Delta^{2}} \cos (k)$ with $\Delta=t / 2$. The spectrum with $\mathrm{PBC}$ is complex for any nonzero $\Delta$, indicating parametric instability. In contrast, the system with $\mathrm{OBC}$ is stable as long as $t>\Delta$, regardless of system size.

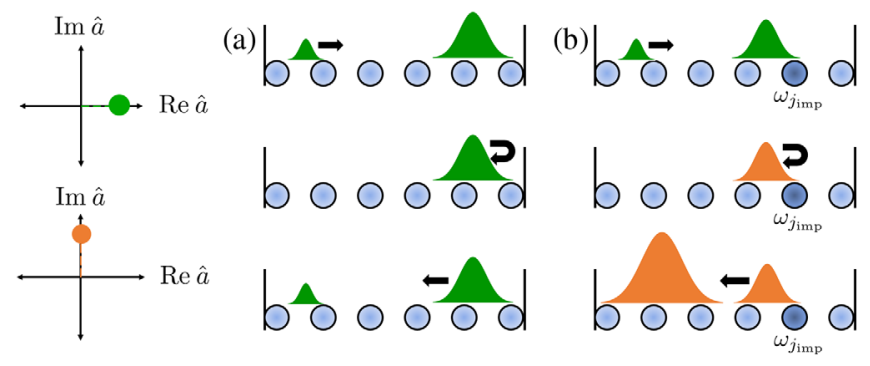

FIG. 4. (a) An $x$ excitation is amplified as it propagates to the right. After being reflected off the boundary, it remains an $x$ excitation as it propagates to the left while being deamplified. The scattering mechanism is the same if we consider reflecting off of on-site loss. (b) An $x$ excitation can turn into a $p$ excitation after scattering off an impurity (dark blue site). In combination with the chiral nature of amplification, multiple reflections between impurities can lead to instability.

$$
\begin{aligned}
& u_{n}(j)=\sqrt{\frac{2}{N+1}} i^{-j} \sin \left(k_{n} j\right) \cosh \left[r\left(j-j_{0}\right)\right], \\
& v_{n}(j)=\sqrt{\frac{2}{N+1}} i^{-j} \sin \left(k_{n} j\right) \sinh \left[r\left(j-j_{0}\right)\right],
\end{aligned}
$$

where the squeeze parameter $r$ is defined in Eq. (8). Note that the chiral symmetry of our system implies that there are many possible choices of eigenmode basis; this choice corresponds to the freedom to pick the parameter $j_{0}$ defining our squeezing transformation.

Equations (22) and (23) show that both the particle and antiparticle parts of the mode wave function are localized: They have an exponential dependence on position, and their weight is concentrated at the ends of the chain. Further, it is the squeezing parameter $r$ which plays the role of an inverse localization length. We note that the parametric dependence of this localization is identical to that in the fermionic Kitaev chain at zero chemical potential. The fermionic model in this case is always topological, and the accompanying Majorana zero-energy edge modes have an inverse localization length given by $r$ as defined in Eq. (8) [1].

Despite the localization of particle and hole wave functions, note that for a given eigenmode, the total contribution of each site to the symplectic norm $\left|u_{n}(j)\right|^{2}-$ $\left|v_{n}(j)\right|^{2}$ does not exhibit any sort of localization. This quantity is, in fact, completely independent of the twophoton driving amplitude $\Delta$. This is consistent with the local Green's function being phase insensitive and independent of $r$. The upshot is that it is difficult to detect the localization of mode wave functions in our system using purely local probes; one must instead consider a nonlocal probe such as transmission.

For an intuitive picture of our model's striking sensitivity to the presence of edges, we return to our picture of chiral wave-packet dynamics. Recall that, e.g., an $x$ quadrature 
excitation propagates to the right with amplification and to the left with deamplification. Thus, in a ring geometry, an initial $x$ excitation can propagate and be amplified indefinitely as it traverses the ring several times in a clockwise direction. This infinite amplification directly leads to dynamical instability. In contrast, in a finite chain, the disturbance will eventually hit the right boundary of the system and be reflected. As it now propagates to the left, it will be deamplified. There is thus no possibility for indefinite amplification, and the system remains stable (see Fig. 4).

\section{EFFECTS OF ON-SITE PERTURBATIONS}

We now explore the sensitivity of our lattice to various types of perturbations, focusing on the case of an open chain with $N$ sites. The simplest kind of perturbation in our model is random on-site losses $\kappa_{j}$. We have already seen that such perturbations are innocuous: While random loss breaks translational invariance and causes reflections within the chain, it never causes any instability. The system remains unitarily equivalent to a simple tight-binding chain with disordered loss; cf. Eqs. (14) and (15).

The situation is markedly different if we have nonzero on-site energies described by

$$
\hat{H}_{\mathrm{dis}}=\sum_{j=1}^{N} \omega_{j} \hat{a}_{j}^{\dagger} \hat{a}_{j} .
$$

Such detuning perturbations can induce instability in our system even if $t>\Delta$. Formally, this instability can be understood from the local squeezing transformation in Eq. (9) that maps our system onto a simple tight-binding chain. While on-site loss terms are invariant under this mapping, on-site detuning terms transform to particlenonconserving parametric drive terms, i.e.,

$$
\begin{aligned}
\hat{U} \hat{H}_{\mathrm{dis}} \hat{U}^{\dagger}= & \sum_{j=1}^{N} \omega_{j}\left(\cosh \left[2 r\left(j-j_{0}\right)\right] \hat{\tilde{a}}_{j}^{\dagger} \hat{\tilde{a}}_{j}\right. \\
& \left.+\frac{1}{2} \sinh \left[2 r\left(j-j_{0}\right)\right]\left[\hat{\tilde{a}}_{j}^{\dagger} \hat{\tilde{a}}_{j}^{\dagger}+\hat{\tilde{a}}_{j} \hat{\tilde{a}}_{j}\right]\right),
\end{aligned}
$$

where we throw away terms proportional to the identity. It is thus generically impossible to transform to a frame where our system conserves particle number, and thus, one can generically get dynamical instability. Note that in the special case where there is just a single impurity at site $j=j_{\text {imp }}$, the system is still always stable: Formally, we could pick the origin $j_{0}$ of our squeezing transformation to coincide with $j_{\text {imp }}$, and thus map our system onto a particleconserving model, which is no longer the case if we were to add even one additional impurity at any other lattice site.

For a more heuristic understanding of how impurities cause instability, we return to the equations of motion for local quadratures. With the relevant perturbations, they now read

$$
\begin{aligned}
& \dot{\hat{x}}_{j}=\frac{\tilde{t}}{2}\left(e^{r} \hat{x}_{j-1}-e^{-r} \hat{x}_{j+1}\right)-\frac{1}{2} \kappa_{j} \hat{x}_{j}+\omega_{j} \hat{p}_{j}, \\
& \dot{\hat{p}}_{j}=\frac{\tilde{t}}{2}\left(e^{-r} \hat{p}_{j-1}-e^{r} \hat{p}_{j+1}\right)-\frac{1}{2} \kappa_{j} \hat{p}_{j}-\omega_{j} \hat{x}_{j} .
\end{aligned}
$$

We see that while loss never dynamically couples $x$ and $p$ quadratures, the same is not true of detuning perturbations. Scattering off impurities changes the phase of an excitation (i.e., converts $x$ to $p$ and vice versa). The chiral nature of amplification in our lattice implies that multiple scatterings of this type can lead to indefinite amplification and thus instability (see Fig. 4). This type of scattering is in contrast to scattering off of local on-site loss or off the boundaries of a finite-sized system, processes which manifestly preserve the excitation's phase and hence do not lead to any instability.

To quantitatively assess the impact of detuning perturbations, we numerically perform a disorder average. We take the $\omega_{j}$ in Eq. (24) to be independent random variables drawn from a uniform distribution on the interval $[-W, W]$, with $W$ representing the disorder strength. While multiple scatterings can lead to instability if there is significant amplification, disorder effects should be weak if $W \ll \tilde{t}$ and $r, N$ are not too large. This intuitive reasoning is borne out by our simulations. Figure 5 shows the results for disorderaveraged scattering probabilities $\left(10^{4}\right.$ realizations $)$ for a

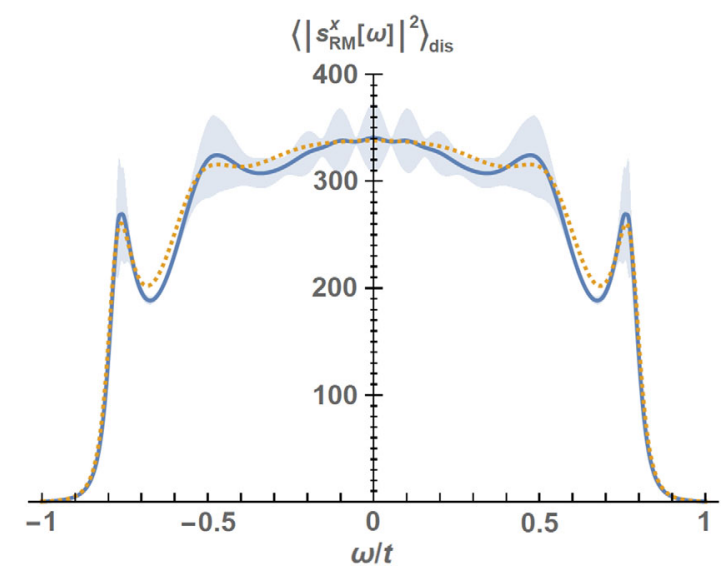

FIG. 5. Disorder-averaged transmission coefficient $\left\langle\left|s_{\mathrm{RM}}^{x}[\omega]\right|^{2}\right\rangle_{\mathrm{dis}}$ for the same setup as is Fig. 2 but with on-site disordered detuning perturbations (disorder strength $W=10^{-3} t$ ). The shaded region corresponds to the variance, and the dashed orange line corresponds to the clean system. Although stability is no longer guaranteed after introducing the detuning perturbations, with the chosen parameters, instability occurs only in less than $0.01 \%$ of realizations. For smaller values of $r$ and/or $N$, one can tolerate even larger amounts of disorder. Parameters: $N=13$ sites, $\Delta=t / 2$, uniform on-site internal loss $\kappa=10^{-2} t$, waveguide coupling rates $\kappa_{M}=2 \kappa_{L}=2 \kappa_{R}=2 t$. 
chain similar to that in Fig. $2(N=13, r \approx 0.55)$ but with disorder strength $W=10^{-3} t$. For these parameters, less than $0.01 \%$ of disorder realizations yield instability. In the remaining instances, the scattering closely resembles the behavior of the clean system.

\section{GENERATION OF MULTIPARTITE ENTANGLEMENT}

We now consider our system's ability to emit entangled photons. Consider the case where $M \leq N$ sites of the lattice are coupled to input-output waveguides. Even if simple vacuum noise is incident in each of these channels, the outgoing light will have nonzero photon number and will exhibit entanglement.

While entanglement generation is generic to any sort of bosonic quantum amplifier, our system has unique features. In particular, the state of the output light has a remarkably transparent form: It is identical to sending a product of single-mode squeezed states into a beam-splitter network, with the squeezing parameters and beam-splitter unitary being directly determined (in a simple way) by the Hamiltonian. This setup is depicted in Fig. 6 and follows immediately from Eqs. (17) and (18). First, one prepares $M$ single-mode squeezed states (one for each channel), with squeeze parameter $R_{j}=r j$ [cf. Eq. (8)] in the waveguide coupled to site $j$. Therefore each channel is populated with photons but there are no correlations. Next, the resulting state is sent into an effective beam-splitter network described by an $M \times M$ unitary matrix $K$, the scattering matrix of a (particle-conserving) tight-binding model with hopping i $\tilde{t}$ [cf. Eq. (11)] and waveguide couplings $\kappa_{j}$. Finally, we apply another set of local, single-mode squeezing transformations on each channel. As this operation is a

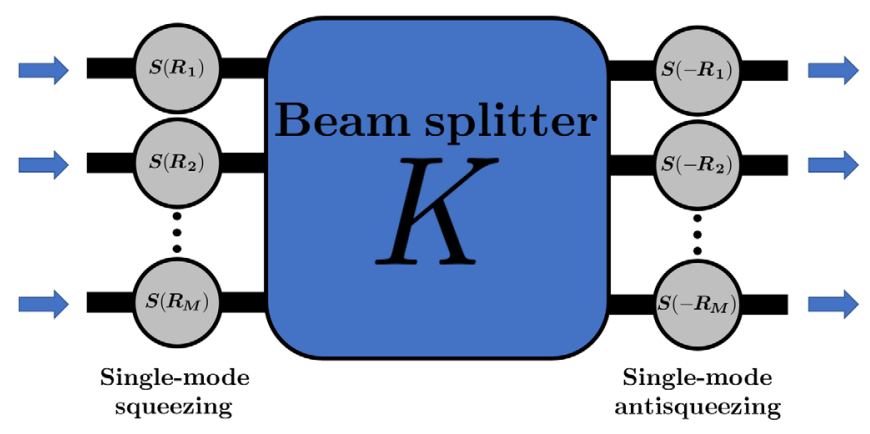

FIG. 6. Schematic showing an equivalent depiction of scattering off our lattice with $M$ input-output waveguides coupled to $M \leq N$ arbitrary sites. Input states in each waveguide are first locally squeezed (squeeze parameters $R_{j}$ ). They then pass through a beam-splitter network and are then finally locally antisqueezed at the outputs. Crucially, the squeeze parameters $R_{j}$ and beam-splitter unitary matrix $K$ have a direct and simple relation to the system Hamiltonian. In particular, $K$ is the unitary of a simple tight-binding model [see Eqs. (E5) and (E6)]. product of purely local unitaries, this last step does not modify entanglement properties.

Note that the resource complexity of such states can be quantified by the number of independent single-mode squeezed states required for their production [25]; in our case, this number is $M$ and can be extremely large. The standard method for producing them requires the experimentally challenging task of first preparing $M$ squeezed states, then transporting and injecting them with high efficiency into a complex beam-splitter network. Our system allows one to circumvent these difficulties by having all the required squeezing generated locally.

States of the form depicted in Fig. 6 have received considerable recent attention. They are of interest as a means to demonstrate "quantum supremacy" [26]. Computing their photon statistics (i.e., Gaussian boson sampling [27]) requires calculating the Halfnian of an $M \times M$ matrix, something that is known to be computationally hard classically (being in the $\sharp P$ complexity class). Output states of this form are also a resource for simulating molecular vibronic spectra [18] and for solving certain classically hard graph-theoretic problems [19].

For applications, one ideally wants the ability to realize a variety of beam-splitter operations $K$ appearing in Fig. 6 . The requisite tunability can be achieved by allowing the magnitude of the hopping and parametric driving on each bond to vary, i.e., $t \rightarrow t_{j}, \Delta \rightarrow \Delta_{j}$. As shown in Appendix E, as long as $\Delta_{j}<t_{j}$ on each bond, our system remains dynamically stable, and the scattering continues to correspond to the schematic in Fig. 6. Now, however, the beam-splitter unitary $K$ corresponds to the scattering matrix of a nonuniform tight-binding chain with hoppings $\tilde{t}_{j}=\sqrt{t_{j}^{2}-\Delta_{j}^{2}}$. Thus, in realizations of our model where one can control $t_{j}, \Delta_{j}$, one has the ability to realize a wide class of nontrivial multimode entangled output states.

\section{CONNECTIONS TO NON-HERMITIAN MODELS}

It is natural to ask whether the striking features of our bosonic model can be given a topological underpinning. To address this, we first make the simple observation that while our Hamiltonian is clearly Hermitian, the mode eigenvalues follow from diagonalizing the system's dynamical matrix [e.g., $\check{M}_{k}$ in momentum space; cf. Eq. (20)]. This matrix is explicitly non-Hermitian. As such, there is an intimate connection between our model (and parametrically driven bosonic systems in general) and the study of non-Hermitian quantum models. Most work on this subject has focused on quadratic Hamiltonians whose corresponding dynamics are simply linear. Since this is also the case here, any interesting physics that can be uniquely attributed to non-Hermicity in these models must also be present in ours. Consequently, recently developed 
topological invariants for non-Hermitian systems can be directly applied to our system.

Note that the dynamical matrix $\check{M}_{k}$ [cf. Eq. (20)] of our model is unitarily equivalent to the non-Hermitian Hamiltonian:

$$
\begin{aligned}
\check{H}_{\mathrm{HN}} & =t \sin (k) \check{1}+i \Delta \cos (k) \check{\sigma}_{z} \\
& =\frac{-i}{2}\left(\begin{array}{cc}
t_{-} e^{i k}-t_{+} e^{-i k} & 0 \\
0 & t_{+} e^{i k}-t_{-} e^{-i k}
\end{array}\right),
\end{aligned}
$$

where $t_{ \pm} \equiv t \pm \Delta$. This non-Hermitian Hamiltonian describes two decoupled 1D chains with asymmetric nearest-neighbor hopping, i.e., two copies of the wellknown Hatano-Nelson model [17]. One chain has stronger left-to-right hopping, while the other has stronger right-toleft hopping. These two effective chains correspond directly to the chirality of quadrature propagation as described by Eqs. (6) and (7).

Non-Hermitian asymmetric hopping models have been the subject of several recent studies exploring topology. In particular, Ref. [28] argues that the winding of the complex spectrum of a single-band 1D non-Hermitian model can be used to define a topological invariant, and that this invariant gives rise to a kind of bulk-boundary correspondence between an infinite system and a semi-infinite system. A nonzero topological number then implies a macroscopic number of edge modes. This has been recently been dubbed the "non-Hermitian skin effect" [29]. This invariant could be applied to each of the decoupled 1D chains described by Eq. (28): The top chain would have a winding +1 and the bottom chain a winding -1 . In another study, Ref. [30] demonstrated that non-Hermitian asymmetric hopping models exhibit striking differences in both their spectrum and wave functions when comparing a ring versus open chain configuration; this is also reminiscent of our model.

Non-Hermitian asymmetric hopping models are often introduced without any clear sense of how they could be physically realized; in addition, any such realization would involve dissipation, and the corresponding fluctuations could disrupt interesting behavior. Our work shows that parametrically driven bosonic systems give a physically realizable platform for a class of effective non-Hermitian models, and moreover, they can do this without any necessity for dissipation and noise.

\section{PHYSICAL REALIZATION}

The parametrically driven coupled-cavity model studied in this work could be realized in a variety of different photonic and phononic systems. $\hat{H}_{B}$ in Eq. (4) describes a 1D array of tunnel-coupled cavities subject to parametric driving on each bond, where we work in a rotating frame at the parametric drive frequency (which is the same for each bond). To construct simple physical implementations of our system, it is easiest to work in a gauge where the
Hamiltonian is real but where the pairing amplitude $\Delta$ is spatially dependent. Making the gauge transformation $\hat{a}_{j} \rightarrow \hat{a}_{j} e^{i \pi j / 2}$ yields

$$
\hat{H}_{B}=\frac{1}{2} \sum_{j}\left(t \hat{a}_{j+1}^{\dagger} \hat{a}_{j}+(-1)^{j} \Delta \hat{a}_{j+1}^{\dagger} \hat{a}_{j}^{\dagger}+\text { H.c. }\right) .
$$

In this gauge, the pairing amplitude phase is modulated from site to site, corresponding to parametric driving where we inject pairs with a center-of-mass momentum $k_{\text {tot }}=\pi$. Note that in this gauge the Hamiltonian is invariant under an inversion operation centered on a bond; this is, however, fully consistent with the chiral propagation we describe (see Appendix F). Our system can thus be realized by using a cavity array with passive nearestneighbor tunneling and nearest-neighbor parametric driving with staggered phases.

A generic implementation is depicted schematically in Fig. 7, where on each link of our main lattice, we have a nonlinear three-wave mixing interaction with an auxiliary mode $\hat{b}_{j}$ :

$$
\hat{H}_{\mathrm{int}}=g_{0} \sum_{j}\left(\hat{a}_{j+1}+\hat{a}_{j+1}^{\dagger}\right)\left(\hat{a}_{j}+\hat{a}_{j}^{\dagger}\right)\left(\hat{b}_{j}+\hat{b}_{j}^{\dagger}\right) .
$$

By coherently driving the $\hat{b}_{j}$ mode with the appropriate frequency and phase, this nonlinear Hamiltonian (within mean-field and rotating-wave approximations) yields the Hamiltonian in Eq. (29), with $\Delta=g_{0}\left|\left\langle\hat{b}_{j}\right\rangle\right|$. Note that pure three-wave mixing elements can be realized in several different ways in superconducting quantum circuits. Examples include the Josephson ring modulator [31] and the recently developed SNAIL Josephson device [32]. Optomechanical systems also feature a nonlinear radiation-pressure coupling between the mechanical and photonic modes [33] and thus present another possible realization.

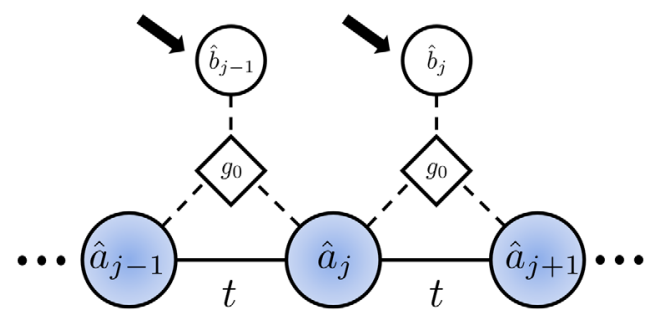

FIG. 7. A possible realization of the bosonic Kitaev-Majorana chain. On each bond, adjacent cavity modes $\hat{a}_{j}$ and $\hat{a}_{j+1}$ are tunnel coupled with hopping amplitude $t$. In addition, they are both coupled to a coherently driven auxiliary mode $\hat{b}_{j}$ via a threewave mixing interaction [Eq. (30)]. At the mean-field level, this interaction yields the parametric two-photon drive in our model. Staggering the drive phases results in a Hamiltonian that is gauge equivalent to a purely imaginary hopping phase, a key ingredient in our setup. 
(a)

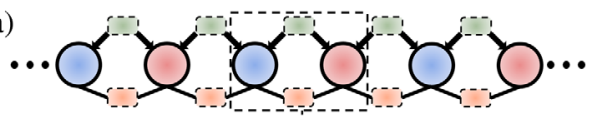

(b)

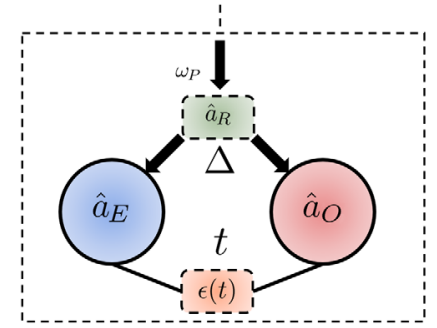

FIG. 8. (a) Schematic of a realization of our model which uses purely intracavity nonlinearities. There are two lattice sites per unit cell, consisting of even and odd lattice sites which have different resonant frequency $\omega_{E}$ and $\omega_{O}$, respectively. Each site is tunnel coupled to four auxiliary resonators. (b) Single unit cell of our 1D lattice. One of the auxiliary cavities has a Kerr-type nonlinearity and is driven nonresonantly at some carefully chosen frequency $\omega_{P}$, which induces the nearest-neighbor parametric drive $\Delta$ on the main lattice sites. Dynamically modulating the dielectric constant of the second auxiliary resonator $\epsilon(t) \propto$ $\cos \left[\left(\tilde{\omega}_{E}-\tilde{\omega}_{O}\right) t\right]$ gives rise to an effective nearest-neighbor hopping $t$ [35-37]. Here, $\tilde{\omega}_{E}$ and $\tilde{\omega}_{O}$ are detuned cavity frequencies; see Appendix $G$ for details.

It is also possible to realize our model [as written in Eq. (29)] using purely intracavity nonlinearities as opposed to nonlinear couplings. This could allow an implementation using, e.g., an array of silicon ring resonators and the intrinsic $\chi^{(3)}$ optical nonlinearity of silicon (as was recently used in Ref. [34] to explore the interplay of parametric driving and topological band structures). The setup in this case is shown in Fig. 8. We take the even and odd cavities of the lattice to have different frequencies $\omega_{E}$ and $\omega_{O}$. Further, on each bond between the primary sites of the lattice, we have two auxiliary cavities. One is driven nonresonantly at $\left(\omega_{E}+\omega_{O}\right) / 2$; using its intrinsic Kerr nonlinearity, the drive realizes the desired nearest-neighbor parametric drive $\Delta$ on the main lattice sites. The second auxiliary cavity supports resonances at both $\omega_{E}$ and $\omega_{O}$; by using dynamic modulation [35-37] (e.g., via an electrooptic modulator), this auxiliary cavity can mediate the hopping $t$ of our model. More details are given in Appendix G.

\section{CONCLUSIONS}

In this work, we introduce and analyze a bosonic version of the well-known fermionic Kitaev chain. Our system does not require strong photon-photon interactions but instead exploits the presence of nontrivial phases in a quadratic, particle-nonconserving Hamiltonian. It exhibits a spatially asymmetric coupling between local quadrature operators, which, in turn, results in a variety of remarkable features. This includes phase-dependent chiral propagation and a striking sensitivity to the presence of edges impacting both the localization and dynamic stability of system eigenmodes. Further, despite being nondissipative, our system has a direct connection to non-Hermitian asymmetric hopping models, which allows us to give our model a topological underpinning.

In terms of outlook, the physics we discuss here could be exploited for applications. Phase-sensitive quantum amplifiers play a central role in several diverse areas of physics such as continuous-variable quantum computing [25] and quantum sensing (see, e.g., Refs. [38,39] for applications to axion detection). As we discuss, our system can serve as a unique kind of phase-sensitive amplifier, in which orthogonal quadratures are amplified in opposite directions. It also is a unique tool for preparing entangled multimode Gaussian states which have recently been proposed as a means to demonstrate quantum supremacy [26]. Our model circumvents the normally difficult task of producing and transporting a large number of squeezed states by generating all squeezing locally.

Our work also suggests directions for future basic research. We obtain unusual physics by replacing fermionic Majorana modes by bosonic quadrature operators: It is only natural to ask whether this can be taken further. The quadrature pairing structure is ultimately what gives rise to the physics we discuss. Future work could thus investigate the effects of dimensionality on this structure, where there is more than right and left hopping, or how symmetries restrict the kind of coupling that the quadratures can take. The role of true photon-photon interactions in this setting would also be extremely interesting.

\section{ACKNOWLEDGMENTS}

We thank Kero Lau for useful conversations. We acknowledge support from NSERC's CGSM program (A. M.), NSERC (T. P. B.), FQRNT (T. P. B.), the Simons Foundation (Grant No. 505450, A.C.), and the NSFMRSEC Grant No. 1420709 (A. M. and A. C.).

\section{APPENDIX A: REAL-VALUED HOPPING}

In this Appendix, we demonstrate that the same model as in Eq. (4) without any hopping phase is trivial. For the case of an infinite lattice, the Heisenberg equations of motion for the momentum space operators are

$$
\begin{gathered}
\left(i \partial_{t}-\check{M}_{k}\right)\left(\begin{array}{c}
\hat{a}_{k} \\
\hat{a}_{-k}^{\dagger}
\end{array}\right)=\left(\begin{array}{l}
0 \\
0
\end{array}\right), \\
\check{M}_{k}=t \cos (k) \check{\sigma}_{z}+i \Delta \cos (k) \check{\sigma}_{x} .
\end{gathered}
$$

The mode energies are readily obtained from the eigenvalues of the dynamical matrix $\check{M}_{k}$ and are given by $E_{k, \pm}= \pm \sqrt{t^{2}-\Delta^{2}} \cos (k)$. The condition for stability $t>\Delta$ is thus the same throughout the band. 
To understand why this model displays trivial physics, we now consider a finite chain of $N$ sites with open boundaries. The Hamiltonian is

$$
\hat{H}_{B}=\frac{1}{2} \sum_{j=1}^{N-1}\left(t \hat{a}_{j+1}^{\dagger} \hat{a}_{j}+i \Delta \hat{a}_{j+1}^{\dagger} \hat{a}_{j}^{\dagger}+\text { H.c. }\right) .
$$

With translational invariance being broken by the boundary, momentum is not conserved, and we can no longer diagonalize the Hamiltonian by moving to a basis of plane waves. Instead, the Hamiltonian is diagonal in the basis of standing waves, which are simply a linear combination of plane waves

$\hat{H}_{B}=\sum_{n=1}^{N}\left[t \cos k_{n} \hat{a}_{k_{n}}^{\dagger} \hat{a}_{k_{n}}+i \frac{\Delta}{2} \cos k_{n}\left(\hat{a}_{k_{n}}^{\dagger} \hat{a}_{k_{n}}^{\dagger}-\right.\right.$ H.c. $\left.)\right]$,

with $\hat{a}_{k_{n}}$ a standing-wave annihilation operator

$$
\hat{a}_{k_{n}}=\sqrt{\frac{2}{N+1}} \sum_{j=1}^{N} \sin \left(k_{n} j\right) \hat{a}_{j},
$$

and $k_{n}=n \pi /(N+1)$. The finite chain with openboundary conditions is essentially no different from the infinite lattice or the chain with periodic boundary conditions. One readily sees that stability is achieved throughout the band if and only if $t>\Delta$, just like in the system without boundaries. Furthermore, the eigenstates of the finite-sized system are standing waves and thus do no exhibit any localization, unlike the model presented in the main text [cf. Eqs. (22) and (23)].

\section{APPENDIX B: ARBITRARY HOPPING PHASE}

Here, we study a similar Hamiltonian to that in Eq. (4), except now the hopping matrix element has an arbitrary phase $i t \rightarrow e^{i \phi} t$. As in the main text, we assume that $t>\Delta$. We find that depending on the magnitude of the real and imaginary part of the hopping, this more general model exhibits similar physics either to the one presented in the main text or a trivial tight-binding model. We explain further in what follows.

First, let us assume that $t|\cos (\phi)|<\Delta$. One can easily verify that

$$
\begin{aligned}
\hat{H}_{B} & =\frac{1}{2} \sum_{j}\left(e^{i \phi} t \hat{a}_{j+1}^{\dagger} \hat{a}_{j}+i \Delta \hat{a}_{j+1}^{\dagger} \hat{a}_{j}^{\dagger}+\text { H.c. }\right) \\
& =\frac{1}{2} \sum_{j}\left(i t^{\prime} \hat{\beta}_{j+1}^{\dagger} \hat{\beta}_{j}+i \Delta^{\prime} \hat{\beta}_{j+1}^{\dagger} \hat{\beta}_{j}^{\dagger}+\text { H.c. }\right),
\end{aligned}
$$

where $t^{\prime}=t \sin (\phi)$ and $\Delta^{\prime}=\sqrt{\Delta^{2}-t^{2} \cos ^{2}(\phi)}$. The $\hat{\beta}_{j}$ are Bogoliubov modes of the original photonic operators

$$
\hat{\beta}_{j}=\cosh (\xi) \hat{a}_{j}+i \sinh (\xi) \hat{a}_{j}^{\dagger},
$$

and the squeeze parameter $\xi$ is defined via

$$
\tanh (2 \xi)=\frac{t \cos (\phi)}{\Delta} .
$$

This is exactly equivalent to the model presented in the main text with renormalized parameters $t \rightarrow t \sin (\phi)$ and $\Delta \rightarrow \sqrt{\Delta^{2}-t^{2} \cos ^{2}(\phi)}$.

On the other hand, if $t|\cos (\phi)|>\Delta$, the previous squeezing transformation is not well defined. We can no longer map our Hamiltonian to a similar model with purely imaginary hopping phase and renormalized parameters. What we can do instead, however, is make a similar transformation that maps the Hamiltonian onto a simple tight-binding model. More concretely, we have

$$
\begin{aligned}
\hat{H}_{B} & =\frac{1}{2} \sum_{j}\left(e^{i \phi} t \hat{a}_{j+1}^{\dagger} \hat{a}_{j}+i \Delta \hat{a}_{j+1}^{\dagger} \hat{a}_{j}^{\dagger}+\text { H.c. }\right) \\
& =\frac{1}{2} \sum_{j}\left(e^{i \tilde{\phi} \tilde{t} \hat{\alpha}_{j+1}^{\dagger}} \hat{\alpha}_{j}+\text { H.c. }\right)
\end{aligned}
$$

where, as in the main text, $\tilde{t}=\sqrt{t^{2}-\Delta^{2}}$. Here we introduce the operators $\hat{\alpha}_{j}$, which are also Bogoliubov modes of the original photonic operators

$$
\hat{\alpha}_{j}=\cosh (\rho) \hat{a}_{j}+i \sinh (\rho) \hat{a}_{j}^{\dagger},
$$

with $\rho$ the squeeze parameter defined via

$$
\tanh (2 \rho)=\frac{\Delta}{t \cos (\phi)},
$$

and $\tilde{\phi}$ is the phase

$$
e^{i \tilde{\phi}}=\frac{i t \sin (\phi)+\operatorname{sgn}[\cos (\phi)] \sqrt{t^{2} \cos ^{2}(\phi)-\Delta^{2}}}{\sqrt{t^{2}-\Delta^{2}}} .
$$

To see why this model does not display any interesting physics, we note that the transformation defined in Eq. (B5) is valid regardless of boundary conditions. Thus, in the regime $t|\cos (\phi)|>\Delta$, one can always map the system onto a simple particle-conserving tight-binding model. This is in contrast with the model considered in the main text which is unitarily equivalent to an excitation-conserving Hamiltonian only in the case of open-boundary conditions. Furthermore, the unitary operator is a local positiondependent squeezing transformation [cf. Eq. (9)], whereas here the relevant operator is spatially uniform. 


\section{APPENDIX C: FINITE CHAIN WITH EDGES: THE $t<\Delta$ CASE}

We now show when $t<\Delta$ the finite chain with openboundary conditions studied in the main text, as defined by Eq. (5), is dynamically unstable. Defining the parameter $\tilde{r}$ via

$$
e^{2 \tilde{r}}=(\Delta+t) /(\Delta-t),
$$

we consider a position-dependent local squeezing transformation defined by

$$
\hat{V} \hat{x}_{j} \hat{V}^{\dagger}=e^{\tilde{r}\left(j-j_{0}\right)} \hat{\tilde{x}}_{j}, \quad \hat{V} \hat{p}_{j} \hat{V}^{\dagger}=e^{-\tilde{r}\left(j-j_{0}\right)} \hat{\tilde{p}}_{j} .
$$

As in the main text, $\hat{\tilde{x}}_{j}, \hat{\tilde{p}}_{j}$ are new canonical quadratures, and $j_{0}$ is an arbitrary real number. One finds that

$$
\begin{aligned}
\hat{V} \hat{H}_{B} \hat{V}^{\dagger} & =\frac{\tilde{\Delta}}{2} \sum_{j}\left(\hat{\tilde{x}}_{j+1} \hat{\tilde{p}}_{j}+\hat{\tilde{p}}_{j+1} \hat{\tilde{x}}_{j}\right) \\
& =\frac{1}{2} \sum_{j}\left(i \tilde{\Delta} \hat{\tilde{a}}_{j+1}^{\dagger} \hat{\tilde{a}}_{j}^{\dagger}+\text { H.c. }\right),
\end{aligned}
$$

with

$$
\tilde{\Delta}=\sqrt{\Delta^{2}-t^{2}}=\Delta / \cosh (\tilde{r}) .
$$

Thus, in the regime $t<\Delta$, the system is unitarily equivalent to a model where we have only pairing (i.e., parametric driving) on each bond with no hopping. It is now evident that the system is dynamically unstable: In this frame, we are resonantly adding pairs of photons on neighboring sites.

\section{APPENDIX D: SCATTERING MATRIX OF A REGULAR TIGHT-BINDING CHAIN}

For completeness, here we derive the expression of the photon scattering matrix of a regular tight-binding chain $\tilde{s}_{j j^{\prime}}[\omega]$, from which we immediately obtain the quadrature scattering matrices; cf. Eqs. (17) and (18). The first step is to compute the Green's function $\tilde{\mathbf{G}}_{0}[\omega]$ of the unperturbed system, i.e., without the spatially dependent loss. By definition,

$$
\tilde{\mathbf{G}}_{0}[\omega]=\left[\left(\omega+i \frac{\kappa}{2}\right) \mathbf{1}-\mathbf{H}\right]^{-1}
$$

where $\kappa$ is the uniform on-site decay rate, and $\mathbf{H}$ is the single-particle Hamiltonian which, in real space, has matrix elements [cf. Eq. (10)]

$$
H_{i j}=i \frac{\tilde{t}}{2} \delta_{i, j+1}-i \frac{\tilde{t}}{2} \delta_{i, j-1} .
$$

Note that we could make a local gauge transformation to make the Hamiltonian matrix $\mathbf{H}$ real valued. However, this transformation would also alter the scattering matrix, and we therefore choose to keep the imaginary phase factors in the definition of the Hamiltonian. With Eq. (D1) in combination with Eq. (D2), one easily verifies that the Green's function for a finite chain of $N$ sites is

$\tilde{G}_{0}\left[j, j^{\prime} ; \omega\right]$

$=i^{j-j^{\prime}} \frac{2 \sin \left\{q[\omega] \min \left(j, j^{\prime}\right)\right\} \sin \left\{q[\omega]\left[N+1-\max \left(j, j^{\prime}\right)\right]\right\}}{\tilde{t} \sin (q[\omega]) \sin \{q[\omega](N+1)\}}$,

where $q[\omega]$ is the complex wave vector satisfying the dispersion

$$
\omega+i \frac{\kappa}{2}-\tilde{t} \cos (q[\omega])=0 .
$$

The introduction of spatially dependent loss $\kappa_{j}$ introduces an effective imaginary potential $\mathbf{V}$ at each lattice site. In real space, it has matrix elements

$$
V_{i j}=-i \frac{\kappa_{j}}{2} \delta_{i j}
$$

The full Green's function $\tilde{\mathbf{G}}[\omega]$ is given by Dyson's equation

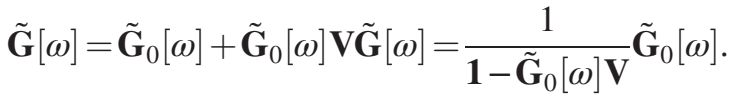

Standard input-output theory $[23,24]$ then gives a simple relation between the scattering matrix and the Green's function

$$
s_{j j^{\prime}}[\omega]=\delta_{j j^{\prime}}-i \sqrt{\kappa_{j} \kappa_{j^{\prime}}} \tilde{G}\left[j, j^{\prime} ; \omega\right] .
$$

\section{APPENDIX E: SPATIALLY VARYING HOPPING AND PAIRING}

We now consider a generalized version of our model where the hopping and parametric drive amplitudes vary from bond to bond:

$\hat{H}_{B^{\prime}} \equiv \frac{1}{2} \sum_{j=1}^{N-1}\left[-\left(t_{j}-\Delta_{j}\right) \hat{x}_{j+1} \hat{p}_{j}+\left(t_{j}+\Delta_{j}\right) \hat{p}_{j+1} \hat{x}_{j}\right]$.

As long as $t_{j}>\Delta_{j}$ for all $j$, the model can still be mapped to a particle-conserving model. Defining

$$
e^{2 r_{j}}=\frac{t_{j}+\Delta_{j}}{t_{j}-\Delta_{j}}, \quad R_{j}=\sum_{m=0}^{j-1} r_{m},
$$


with $r_{0}$ an arbitrary real number, we make a local positiondependent squeezing transformation:

$$
\hat{U} \hat{x}_{j} \hat{U}^{\dagger}=e^{R_{j}} \hat{\tilde{x}}_{j}, \quad \hat{U} \hat{p}_{j} \hat{U}^{\dagger}=e^{-R_{j}} \hat{\tilde{p}}_{j} .
$$

One finds that

$$
\hat{U} \hat{H}_{B^{\prime}} \hat{U}^{\dagger}=\frac{1}{2} \sum_{j}\left(i \tilde{t}_{j} \hat{\tilde{a}}_{j+1}^{\dagger} \hat{\tilde{a}}_{j}+\text { H.c. }\right),
$$

with $\tilde{t}_{j}=\sqrt{t_{j}^{2}-\Delta_{j}^{2}}$.

Thus, in the case where $t_{j}>\Delta_{j}$ for all $j, \hat{H}_{B^{\prime}}$ is unitarily equivalent to a particle-conserving tight-binding chain with a spatially varying tunnel matrix element $i \tilde{t}_{j}$. This mapping also implies a simple form for the scattering matrices that correspond to Fig. 6:

$$
\begin{gathered}
s_{j j^{\prime}}^{x}[\omega]=e^{R_{j}-R_{j^{\prime}}} \tilde{s}_{j j^{\prime}}[\omega], \\
s_{j j^{\prime}}^{p}[\omega]=e^{-\left(R_{j}-R_{j^{\prime}}\right)} \tilde{s}_{j j^{\prime}}[\omega],
\end{gathered}
$$

where now $\tilde{s}_{j j^{\prime}}[\omega]$ is the scattering matrix of an $N$-site tightbinding chain with hopping matrix elements $i \tilde{t}_{j}$ and on-site decay rates $\kappa_{j}$.

\section{APPENDIX F: CHIRALITY IN THE REAL GAUGE}

The Hamiltonian Eq. (29) is invariant under a spatial inversion centered on a bond. This would seem to imply that there can never be any sort of chirality in our model. Yet we know this must be false: The Hamiltonian in the gauge used throughout most of the main text Eq. (5) and the corresponding equations of motion Eqs. (6) and (7) manifestly are. The issue is resolved by defining dynamically decoupled quadratures in a site-dependent manner. Working in the gauge used to write Eq. (29), we define the quadratures of interest differently on even and odd sites. For even $j$, we define

$$
\begin{aligned}
& \hat{x}_{j}=\frac{e^{i \pi j / 2}}{\sqrt{2}}\left(\hat{a}_{j}+\hat{a}_{j}^{\dagger}\right), \\
& \hat{p}_{j}=\frac{e^{i \pi j / 2}}{\sqrt{2} i}\left(\hat{a}_{j}-\hat{a}_{j}^{\dagger}\right),
\end{aligned}
$$

and for odd $j$, we define

$$
\begin{aligned}
& \hat{x}_{j}=\frac{e^{i \pi(j+1) / 2}}{\sqrt{2} i}\left(\hat{a}_{j}-\hat{a}_{j}^{\dagger}\right), \\
& \hat{p}_{j}=\frac{e^{i \pi(j-1) / 2}}{\sqrt{2}}\left(\hat{a}_{j}+\hat{a}_{j}^{\dagger}\right) .
\end{aligned}
$$

One readily verifies that the equations of motion of these new quadrature operators are the same as in the old gauge [i.e., Eqs. (6) and (7)], and all transport properties are therefore identical.

Working in the same gauge, consider a driving force on site 1 that excites the $\hat{x}$ quadrature which propagates and is amplified to the right. One could imagine the corresponding solution to the Schrödinger equation $|\psi(t)\rangle$ that describes this propagation. We may now apply an inversion operation centered on a bond and ask what are the transport properties in this new frame. The Hamiltonian is invariant under such a transformation, whereas the direction of propagation of the state is now to the left. However, inversion centered on a bond exchanges even and odd lattice sites, and consequently, the force in this new frame forces a $\hat{p}$ quadrature. There is thus no contradiction: One must simply be careful in identifying the correct degrees of freedom.

\section{APPENDIX G: PHYSICAL REALIZATION USING INTRACAVITY NONLINEARITIES}

We now demonstrate that our model can be realized using only intracavity nonlinearities, as shown in Fig. 8. Let us first focus on the two lattice sites $E$ and $O$ as well as the driven nonlinear resonator $R$. In the lab frame, the Hamiltonian describing these three elements is

$$
\begin{aligned}
H= & \omega_{E} \hat{a}_{E}^{\dagger} \hat{a}_{E}+\omega_{O} \hat{a}_{O}^{\dagger} \hat{a}_{O}+\omega_{R} \hat{a}_{R}^{\dagger} \hat{a}_{R}+\frac{U}{2} \hat{a}_{R}^{\dagger} \hat{a}_{R}^{\dagger} \hat{a}_{R} \hat{a}_{R} \\
& +J_{E}\left(\hat{a}_{R}^{\dagger} \hat{a}_{E}+\text { H.c. }\right)+J_{O}\left(\hat{a}_{R}^{\dagger} \hat{a}_{O}+\text { H.c. }\right),
\end{aligned}
$$

where $\hat{a}_{R}$ is the nonlinear auxiliary resonator, and $\hat{a}_{E}$ and $\hat{a}_{O}$ are the main modes of our lattice. Moving to a rotating frame with the appropriate unitary $U=$ $\exp \left[i\left(\tilde{\omega}_{E} \hat{a}_{E}^{\dagger} \hat{a}_{E}+\tilde{\omega}_{O} \hat{a}_{O}^{\dagger} \hat{a}_{O}\right) t\right]$, the Hamiltonian becomes

$$
\begin{aligned}
H= & \left(\omega_{E}-\tilde{\omega}_{E}\right) \hat{a}_{E}^{\dagger} \hat{a}_{E}+\left(\omega_{O}-\tilde{\omega}_{O}\right) \hat{a}_{E}^{\dagger} \hat{a}_{E}+\omega_{R} \hat{a}_{R}^{\dagger} \hat{a}_{R} \\
& +\frac{U}{2} \hat{a}_{R}^{\dagger} \hat{a}_{R}^{\dagger} \hat{a}_{R} \hat{a}_{R}+J_{E}\left(e^{-i \tilde{\omega}_{E} t} \hat{a}_{R}^{\dagger} \hat{a}_{E}+\text { H.c. }\right) \\
& +J_{O}\left(e^{-i \tilde{\omega}_{O} t} \hat{a}_{R}^{\dagger} \hat{a}_{B}+\text { H.c. }\right) .
\end{aligned}
$$

Note that the frequencies $\tilde{\omega}_{E}$ and $\tilde{\omega}_{O}$ are not the exact resonance frequencies of $\omega_{E}$ and $\omega_{O}$. As we soon see, we work in this frame to cancel dispersive shifts caused by the resonator mode $\hat{a}_{R}$ on our two main cavity modes.

In this frame, the Heisenberg equations of motion are

$$
\begin{gathered}
\partial_{t} \hat{a}_{R}=-\left(i \omega_{R}+\frac{\kappa_{R}}{2}\right) \hat{a}_{R}-i U \hat{a}_{R}^{\dagger} \hat{a}_{R} \hat{a}_{R} \\
-i J_{E} e^{-i \tilde{\omega}_{E} t} \hat{a}_{E}-i J_{O} e^{-i \tilde{\omega}_{O} t} \hat{a}_{O}, \\
\partial_{t} \hat{a}_{E}=-\left(i\left(\omega_{E}-\tilde{\omega}_{E}\right)+\frac{\kappa_{E}}{2}\right) \hat{a}_{E}-i J_{E} e^{i \tilde{\omega}_{E} t} \hat{a}_{R},
\end{gathered}
$$




$$
\partial_{t} \hat{a}_{O}=-\left(i\left(\omega_{O}-\tilde{\omega}_{O}\right)+\frac{\kappa_{O}}{2}\right) \hat{a}_{O}-i J_{O} e^{i \tilde{\omega}_{O} t} \hat{a}_{R}
$$

We now drive the nonlinear mode with frequency $\omega_{P}$ and write $\hat{a}_{P}=e^{-i \omega_{P} t}\left(\alpha+\delta \hat{a}_{P}\right)$, where $\delta \hat{a}_{P}$ is the deviation from the amplitude $\alpha$ that solves the classical equations of motion. We then linearize the equations of motion by keeping terms that are to first order in $\delta \hat{a}_{R}$,

$$
\begin{aligned}
\partial_{t} \delta \hat{a}_{R}= & -\left(i \tilde{\omega}_{d}+\frac{\kappa_{R}}{2}\right) \delta \hat{a}_{R}-i U \alpha^{2} \delta \hat{a}_{R}^{\dagger} \\
& -i J_{E} e^{-i\left(\omega_{E}-\omega_{P}\right) t} \hat{a}_{E}-i J_{O} e^{-i\left(\omega_{O}-\omega_{P}\right) t} \hat{a}_{O}, \\
\partial_{t} \hat{a}_{E}= & -\left(i\left(\omega_{E}-\tilde{\omega}_{E}\right)+\frac{\kappa_{E}}{2}\right) \hat{a}_{E} \\
& -i J_{E} e^{i\left(\omega_{E}-\omega_{P}\right) t}\left(\alpha+\delta \hat{a}_{R}\right), \\
\partial_{t} \hat{a}_{O}= & -\left(i\left(\omega_{O}-\tilde{\omega}_{O}\right)+\frac{\kappa_{O}}{2}\right) \hat{a}_{O} \\
& -i J_{O} e^{i\left(\omega_{O}-\omega_{P}\right) t}\left(\alpha+\delta \hat{a}_{R}\right),
\end{aligned}
$$

with $\tilde{\omega}_{d}=\omega_{R}-\omega_{P}+2 U|\alpha|^{2}$ the effective detuning. Assuming that the effective detuning $\tilde{\omega}_{d}$ is the largest frequency scale in this problem, we may adiabatically eliminate $\delta \hat{a}_{R}$. Thus, as far as modes $\hat{a}_{E}$ and $\hat{a}_{O}$ are concerned, $\delta \hat{a}_{R}$ has no dynamics, and we may set the time derivative of $\delta \hat{a}_{R}$ to zero. We then express $\delta \hat{a}_{R}$ as a function of $\hat{a}_{E}, \hat{a}_{O}$ and insert that into their respective equations of motion. After doing so, the equations of motion for $\hat{a}_{E}$ now read

$$
\begin{aligned}
\partial_{t} \hat{a}_{E}= & \left(-\frac{\kappa_{A}}{2}-i \tilde{\Omega}_{E}\right) \hat{a}_{E}-i \tilde{\lambda}_{E} e^{2 i\left(\tilde{\omega}_{E}-\omega_{P}\right) t} \hat{a}_{E}^{\dagger} \\
& -i \tilde{J} e^{i\left(\tilde{\omega}_{E}-\tilde{\omega}_{O}\right) t} \hat{a}_{O}-i \Delta e^{i\left(\tilde{\omega}_{E}+\tilde{\omega}_{O}-2 \omega_{P}\right) t} \hat{a}_{O}^{\dagger},
\end{aligned}
$$

where we omit a constant term that oscillates at frequency $\tilde{\omega}_{E}-\tilde{\omega}_{P}$. The equation of motion for $\hat{a}_{O}$ is the same, with $E \leftrightarrow O$. The effective coupling constants are

$$
\begin{gathered}
\tilde{\Omega}_{E}=\omega_{E}-\tilde{\omega}_{E}-J_{E}^{2} \frac{\tilde{\omega}_{d}-i \frac{\kappa_{R}}{2}}{\tilde{\omega}_{d}^{2}+\frac{\kappa_{R}^{2}}{4}-U^{2}|\alpha|^{4}}, \\
\tilde{\lambda}_{E}=J_{E}^{2} \frac{U \alpha^{2}}{\tilde{\omega}_{d}^{2}+\frac{\kappa^{2}}{4}-U^{2}|\alpha|^{4}}, \\
\tilde{J}=-J_{E} J_{O} \frac{\tilde{\omega}_{d}-i \frac{\kappa_{R}}{2}}{\tilde{\omega}_{d}^{2}+\frac{\kappa^{2}}{4}-U^{2}|\alpha|^{4}}, \\
\Delta=J_{E} J_{O} \frac{U \alpha^{2}}{\tilde{\omega}_{d}^{2}+\frac{\kappa^{2}}{4}-U^{2}|\alpha|^{4}} .
\end{gathered}
$$

The pump must be chosen self-consistently to satisfy $2 \omega_{P}=\tilde{\omega}_{E}+\tilde{\omega}_{O}$, and $\Re\left(\tilde{\Omega}_{E}\right)=\Re\left(\tilde{\Omega}_{O}\right)=0$. The first condition asserts that, in the rotating frame, we are resonantly creating pairs of photons in the even and odd cavities. The second condition is required to cancel the AC Stark shifts induced by the resonator mode which is detrimental to the stability of the bosonic KitaevMajorana chain. If we then chose $\tilde{\omega}_{E}$ and $\tilde{\omega}_{O}$ to be very detuned, then all time-dependent terms in Eq. (G9) are nonresonant and drop out under the rotating-wave approximation. We are then simply left with an effective nearestneighbor parametric drive with pairing amplitude $\Delta$.

To implement the nearest-neighbor hopping in our lattice, we must couple the even and odd lattice sites to another auxiliary cavity. This cavity has mode spacing $\tilde{\omega}_{E}-\tilde{\omega}_{O}$. By using two phase modulators oscillating at frequency $\tilde{\omega}_{E}-\tilde{\omega}_{O}$, one can ensure phase matching. The upshot is an effective nearest-neighbor coupling $t$, in which photons can tunnel between the two lattice sites, despite being very separated in energy (see Ref. [37] for details).

[1] A. Kitaev, Unpaired Majorana Fermions in Quantum Wires, Phys. Usp. 44, 131 (2001).

[2] J. Alicea, New Directions in the Pursuit of Majorana Fermions in Solid State Systems, Rep. Prog. Phys. 75, 076501 (2012).

[3] C. W. J. Beenakker, Search for Majorana Fermions in Superconductors, Annu. Rev. Condens. Matter Phys. 4, 113 (2013).

[4] S. D. Sarma, M. Freedman, and C. Nayak, Majorana Zero Modes and Topological Quantum Computation, npj Quantum Inf. 1, 15001 (2015).

[5] Y. Oreg, G. Refael, and F. von Oppen, Helical Liquids and Majorana Bound States in Quantum Wires, Phys. Rev. Lett. 105, 177002 (2010).

[6] T. Karzig, Y. Oreg, G. Refael, and M. H. Freedman, Universal Geometric Path to a Robust Majorana Magic Gate, Phys. Rev. X 6, 031019 (2016).

[7] R. Barnett, Edge-State Instabilities of Bosons in a Topological Band, Phys. Rev. A 88, 063631 (2013).

[8] R. Shindou, R. Matsumoto, S. Murakami, and J.-i. Ohe, Topological Chiral Magnonic Edge Mode in a Magnonic Crystal, Phys. Rev. B 87, 174427 (2013).

[9] B. Galilo, D. K. K. Lee, and R. Barnett, Selective Population of Edge States in a 2D Topological Band System, Phys. Rev. Lett. 115, 245302 (2015).

[10] G. Engelhardt and T. Brandes, Topological Bogoliubov Excitations in Inversion-Symmetric Systems of Interacting Bosons, Phys. Rev. A 91, 053621 (2015).

[11] C.-E. Bardyn, T. Karzig, G. Refael, and T. C. H. Liew, Chiral Bogoliubov Excitations in Nonlinear Bosonic Systems, Phys. Rev. B 93, 020502 (2016).

[12] V. Peano, M. Houde, C. Brendel, F. Marquardt, and A. A. Clerk, Topological Phase Transitions and Chiral Inelastic Transport Induced by the Squeezing of Light, Nat. Commun. 7, 10779 (2016). 
[13] V. Peano, M. Houde, F. Marquardt, and A. A. Clerk, Topological Quantum Fluctuations and Traveling Wave Amplifiers, Phys. Rev. X 6, 041026 (2016).

[14] G. Engelhardt, M. Benito, G. Platero, and T. Brandes, Topological Instabilities in ac-Driven Bosonic Systems, Phys. Rev. Lett. 117, 045302 (2016).

[15] C. E. Bardyn and A. Imamoglu, Majorana-like Modes of Light in a One-Dimensional Array of Nonlinear Cavities, Phys. Rev. Lett. 109, 253606 (2012).

[16] M. Greiter, V. Schnells, and R. Thomale, The ID Ising Model and the Topological Phase of the Kitaev Chain, Ann. Phys. (Amsterdam) 351, 1026 (2014).

[17] N. Hatano and D. R. Nelson, Vortex Pinning and NonHermitian Quantum Mechanics, Phys. Rev. B 56, 8651 (1997).

[18] J. Huh, G. G. Guerreschi, B. Peropadre, J. R. McClean, and A. Aspuru-Guzik, Boson Sampling for Molecular Vibronic Spectra, Nat. Photonics 9, 615 (2015).

[19] K. Brádler, P.-L. Dallaire-Demers, P. Rebentrost, D. Su, and C. Weedbrook, Gaussian Boson Sampling for Perfect Matchings of Arbitrary Graphs, Phys. Rev. A 98, 032310 (2018).

[20] C. L. Kane and E. J. Mele, $Z_{2}$ Topological Order and the Quantum Spin Hall Effect, Phys. Rev. Lett. 95, 146802 (2005).

[21] C. L. Kane and E. J. Mele, Quantum Spin Hall Effect in Graphene, Phys. Rev. Lett. 95, 226801 (2005).

[22] J. Maciejko, T. L. Hughes, and S.-C. Zhang, The Quantum Spin Hall Effect, Annu. Rev. Condens. Matter Phys. 2, 31 (2011).

[23] C. W. Gardiner and P. Zoller, Quantum Noise (Springer, Berlin, 2000).

[24] A. A. Clerk, M. H. Devoret, S. M. Girvin, F. Marquardt, and R.J. Schoelkopf, Introduction to Quantum Noise, Measurement and Amplification, Rev. Mod. Phys. 82, 1155 (2010).

[25] S. L. Braunstein, Squeezing as an Irreducible Resource, Phys. Rev. A 71, 055801 (2005).
[26] S. Aaronson and A. Arkhipov, The Computational Complexity of Linear Optics, Theory Comput. 9, 143 (2013).

[27] C. S. Hamilton, R. Kruse, L. Sansoni, S. Barkhofen, C. Silberhorn, and I. Jex, Gaussian Boson Sampling, Phys. Rev. Lett. 119, 170501 (2017).

[28] Z. Gong, Y. Ashida, K. Kawabata, K. Takasan, S. Higashikawa, and M. Ueda, Topological Phases of NonHermitian Systems, Phys. Rev. X 8, 031079 (2018).

[29] S. Yao and Z. Wang, Edge States and Topological Invariants of Non-Hermitian Systems, Phys. Rev. Lett. 121, 086803 (2018).

[30] Y. Xiong, Why Does Bulk Boundary Correspondence Fail in Some Non-Hermitian Topological Models, J. Phys. Commun. 2, 035043 (2018).

[31] B. Abdo, A. Kamal, and M. Devoret, Nondegenerate Three-Wave Mixing with the Josephson Ring Modulator, Phys. Rev. B 87, 014508 (2013).

[32] N. E. Frattini, U. Vool, S. Shankar, A. Narla, K. M. Sliwa, and M.H. Devoret, 3-Wave Mixing Josephson Dipole Element, Appl. Phys. Lett. 110, 222603 (2017).

[33] M. Aspelmeyer, T. J. Kippenberg, and F. Marquardt, Cavity Optomechanics, Rev. Mod. Phys. 86, 1391 (2014).

[34] S. Mittal, E. A. Goldschmidt, and M. Hafezi, A Topological Source of Quantum Light, Nature (London) 561, 502 (2018).

[35] K. Fang, Z. Yu, and S. Fan, Realizing Effective Magnetic Field for Photons by Controlling the Phase of Dynamic Modulation, Nat. Photonics 6, 782 (2012).

[36] L. Yuan and S. Fan, Bloch Oscillation and Unidirectional Translation of Frequency in a Dynamically Modulated Ring Resonator, Optica 3, 1014 (2016).

[37] L. Yuan, M. Xiao, Q. Lin, and S. Fan, Synthetic Space with Arbitrary Dimensions in a Few Rings Undergoing Dynamic Modulation, Phys. Rev. B 97, 104105 (2018).

[38] Z. Ahmed et al., Quantum Sensing for High Energy Physics, arXiv:1803.11306.

[39] H. Zheng, M. Silveri, R. T. Brierley, S. M. Girvin, and K. W. Lehnert, Accelerating Dark-Matter Axion Searches with Quantum Measurement Technology, arXiv:1607.02529. 